\title{
The Production of Nanoparticles Chitosan from Crustaceans Shell Using the Top-down Method
}

\author{
Syamdidi $^{1 *}$, Helita Sidauruk ${ }^{2}$, Aris Munandar ${ }^{2}$, and Sakinah Haryati ${ }^{2}$ \\ ${ }^{1}$ Research and Development Centre for Marine and Fisheries Product Processing and Biotechnology, \\ Jl. KS. Tubun Petamburan VI, Jakarta, Indonesia \\ ${ }^{2}$ Sultan Ageng Tirtayasa University, Serang, Banten Province, Indonesia
}

\begin{abstract}
Research on the manufacture of nanoparticles of chitosan through top-down method has a limited publication. Most of the research on chitosan nanoparticles was conducted by ionic gelation and other methods. Therefore, this study aimed to investigate the characteristics of nanoparticles chitosan through top-down method. The rprocedure was composed using High Energy Milling (HEM) with different sizes of ball and milling time. The responses observed from this research were particle sizes, yield, viscosity and zeta potential. The results showed that milling time significantly affected the particle size. Milling time between 3 to 5 hour produce the particle size close to $200 \mathrm{~nm}$. Milling duration for 5 hours with a small ball size produced shrimp chitosan nanoparticles with the value of $248.28 \mathrm{~nm}$ particle size, zeta potential $\mathrm{mV}$ of 44.92 , yield of $96 \%$ and $104.88 \mathrm{cP}$ viscosity. The nanoparticle produced was categorized as relatively stable because its zeta potential values were around $\pm 30 \mathrm{mV}$.
\end{abstract}

\section{Introduction}

Chitosan is a chitin derivative compound that has undergone a deacetylation process. Chitosan is a biopolymer that is widely used in various industries as a coagulant in wastewater treatment, metal ion adsorbent, anti-cancer / anti-tumor, and food preservatives $[1,2]$.

Those advantages of chitosan make this polymer globally used as a raw material in many industries, for example in pharmaceutical industry. Chitosan is used as drug carrier due to their ability to reach the side of therapeutic action (3). However, its larger particle limits the utilization and also the ease of chitosan application. So, one of solutions to solve this problem is to reduce the size of the particle into nano size. Nanoparticle chitosan has received great attention because of their nano-size, large surface area, biodegradable, and good biocompatibility [4].

Chitosan nanoparticles are solid colloidal particles with diameters ranging from 1-1000 $\mathrm{nm}$ [3]. The synthesis method for making nanoparticles is divided into two, namely topdown and bottom-up approaches. The top-down approach is to break down large particles

\footnotetext{
* Corresponding author : syamdidi@gmail.om
} 
into nanometer-sized particles while bottom-up is to synthesis the material from atomic size via chemical reaction to produce nanometer-sized particles.

Chitosan nanoparticles increase the advantages of chitosan since more stable, increasing chitosan adsorption power, improving delivery ability [5]. Preparation of chitosan nanoparticles over the past several decades has been carried out by various methods of ionic gelation, microemulsions, emulsification, cross-linking, polymerization. Various differences in this method give different results for particle size and molecular structure of chitosan particles formed. The results from those methods tend to be ranged from $300 \mathrm{~nm}$ $500 \mathrm{~nm}$ [5]. The previous studies of chitosan nanoparticles mostly use bottom-up methods which involve chemical reactions and have not found any nanoparticle preparation with the top-down method on chitosan nanoparticles.

So that, it is necessary to study the preparation of chitosan nanoparticles using the topdown method using mechanics, namely the High Energy Milling (HEM) machine. HEM is a device that uses collision energy between balls and chamber walls which are rotated and moved in a certain way. The advantage of HEM is that it can make nanoparticles in a relatively short time and can make nanoparticles in the desired conditions or atmosphere during the milling process and can also produce relatively large amounts of nanoparticles.

\section{Materials and methods}

\subsection{Materials}

Material used in this study was chitosan from shrimp shell, tween 80 and aquadest. Equipment used were weighing scale, shovel machines, High Energy Milling (PW 1000 DI HEM), milling balls, Particle Size Analyzer (PSA) 2000 MU and Brookfield viscometer DV2T LV.

\subsection{Procedure}

The factors used in this study were the size of the ball (diameter $3 \mathrm{~mm}$ and $6 \mathrm{~mm}$ ) and the milling time used ( 3 hours, 4 hours and 5 hours). Repetition is done 3 times for each treatment. The chitosan powder was put in chamber wall of High Energy Milling Machine according to three different times and ball size. The nanoparticles obtained then analyzed for yields, viscosity by dissolved $1 \mathrm{~g}$ of chitosan in $100 \mathrm{ml}$ acetic acid solution then analyzed using Brookfield DV2T LV model viscometer.(6), particle size (7) and zeta potential (7) determined by putting $0.5 \mathrm{~g}$ chitosan in $100 \mathrm{ml}$ HPLC grade water then added with 2 drops of tween 80 analyzed using particle size analyzer (PSA) 2000 MU.

\section{Result and discussion}

\subsection{Yield}

The yield of shrimp nano chitosan ranged from $95.25 \%-98.01 \%$. The value of the yield of nano chitosan can be seen in Figure 1. 


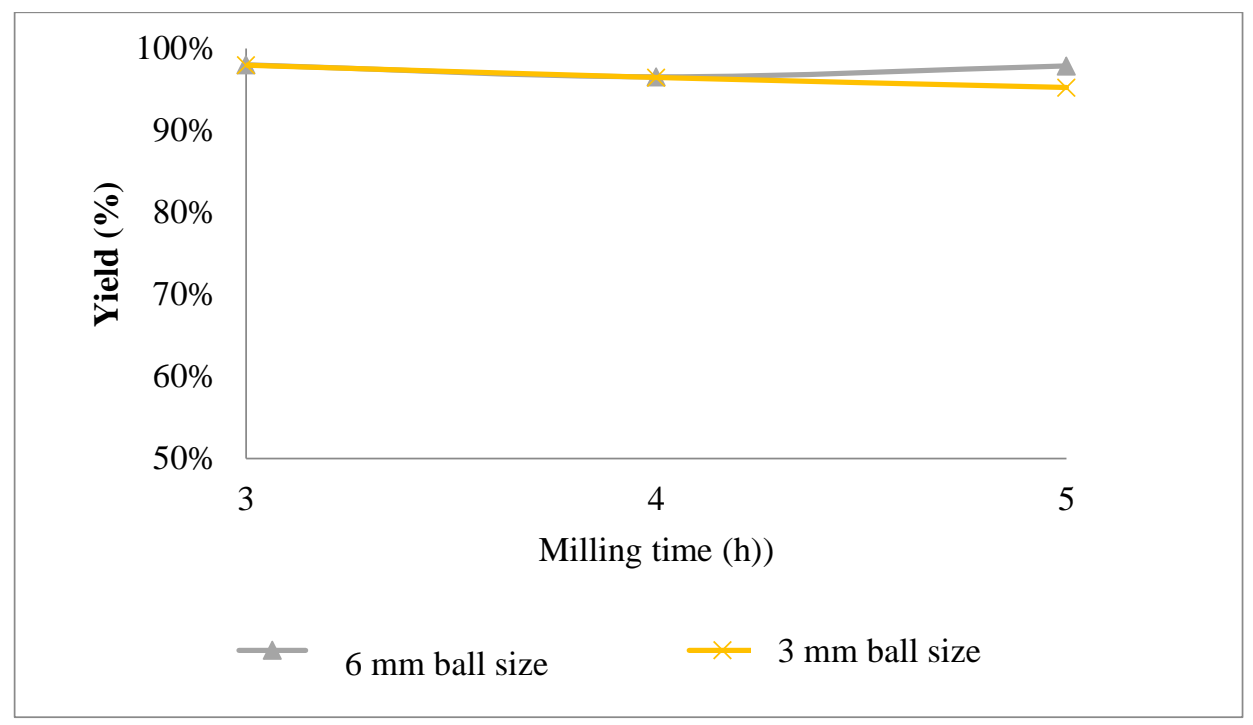

Fig. 1. Yield of nano chitosan at various treatments

The yield of shrimp chitosan nanoparticles at various treatments showed no significant difference. But in general, the yield decreases as the milling time increases for both $3 \mathrm{~mm}$ and $6 \mathrm{~mm}$ ball. This is one of the advantages of the milling method which results in a high yield. This is because the size of the nanoparticles produced is not yet small enough so that it does not decrease much during the sieving process. Some research showed that milling time could be up to 10 hours, longer than milling time in this study [8]. [9] stated that the smaller particle size will reduce its yield because the fraction is light so it is easily wasted. In addition, it was alleged that chitosan nanoparticles produced only experienced a small amount of agglomeration that occurred between the material and the milling ball.

\subsection{Viscosity}

Viscosity is the ability of the fluid to resist friction or displace the layers. The magnitude of force is needed to give rise to a certain velocity associated with the viscosity of a fluid. In different fluids, the more viscous the fluid is, the greater the force required. In liquid, viscosity is caused by cohesion forces between molecules. The viscosity test aims to determine the degree of thickness of the chitosan nanoparticles produced. The viscosity of nano chitosan in various treatments can be seen in Figure 2. 


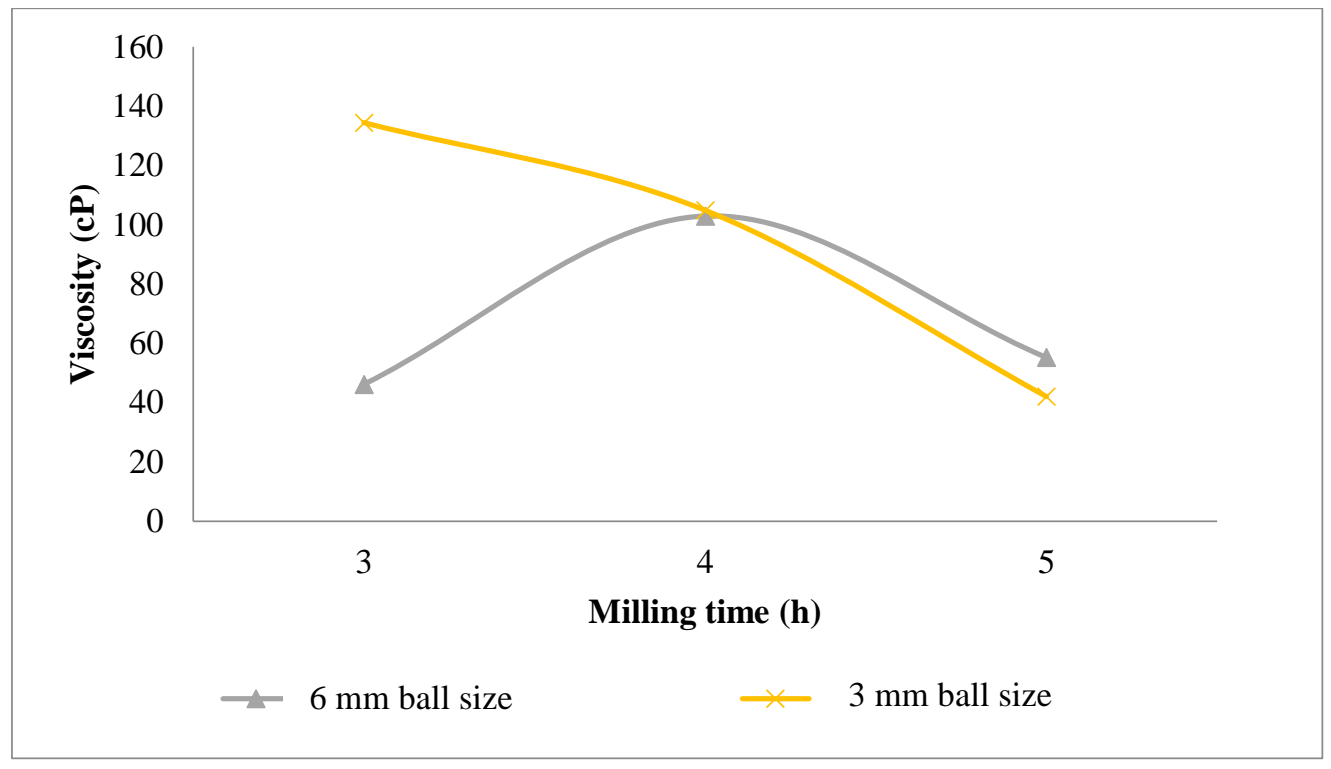

Fig. 2. Viscosity of nano chitosan at various treatments

The picture above shows the viscosity of shrimp chitosan nanoparticles ranging from $42.07 \mathrm{cP}-134.44 \mathrm{cP}$. It can be concluded from Fig. 2 that milling time seems gave significant effect to the viscosity. The longer milling time, the lower viscous the nano chitosan is. The different ball size used did not give significant effect to the viscosity of nano chitosan. The value of viscosity gained is categorized as low viscosity, $<200 \mathrm{cP}(9)$.

This is because the difference in the size of the milling ball and the milling time has not yet produced a small enough size of the particle, so the viscosity value is still low. The smaller particle size causes the surface area to increase so that water absorption is greater. The ability to absorb water itself is very much related to viscosity. The ability to absorb large amounts of water makes the ability to absorb water increase.

\subsection{Particle size}

Nano chitosan produced was tested for its size using a Particle Size Analyzer (PSA). Milling is one of the top down methods of nanoparticle synthesis. Grinding the material from bulk (bulk material) into smaller dimensions causes the particle size of the material to decrease. The particle size of nano chitosan shrimp can be seen in Figure 3. 


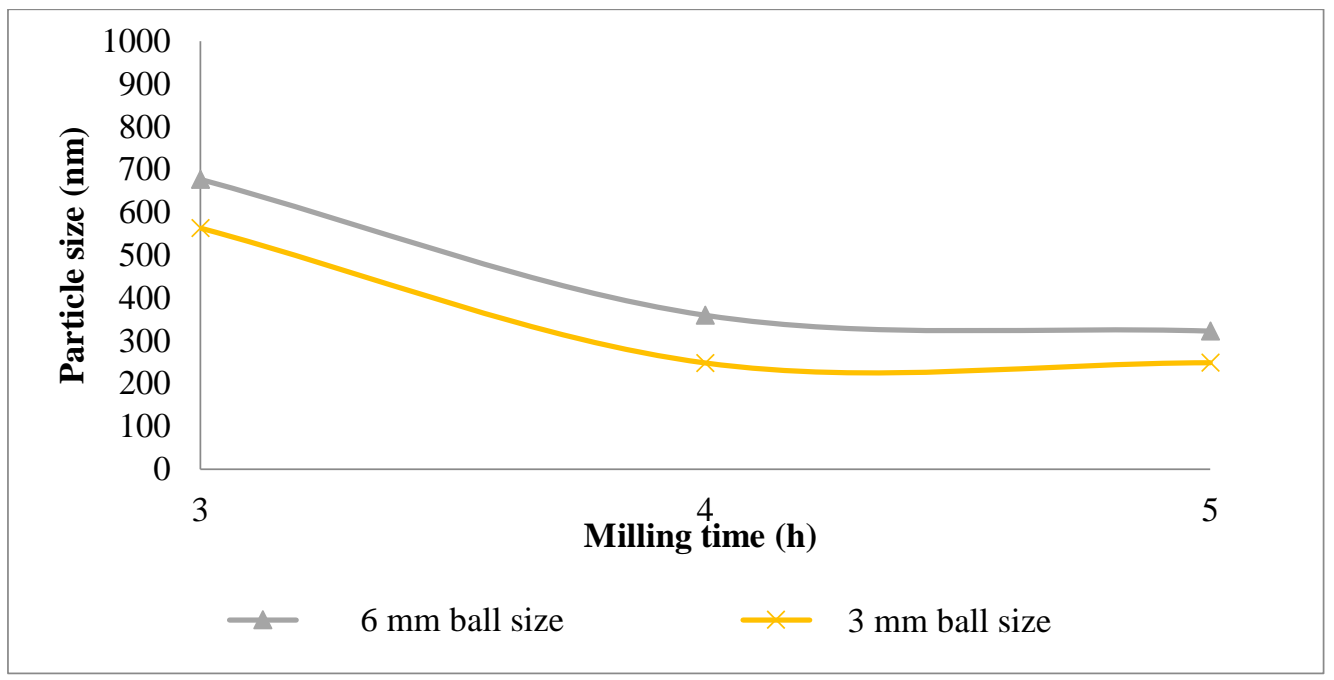

Fig. 3. Particle size of nano chitosan at various treatments

Fig. 3 shows that the particle size of chitosan nanoparticles ranged from $248.84 \mathrm{~nm}$ to $677.16 \mathrm{~nm}$. The particle size distribution test using Particle Size Analyzer showed that particle sizes produced were in the nanoparticle size range. Mohanraj [11] suggested that nanoparticles are solid particles with a size range of 10-1000 nm.

It can be seen in Fig. 3 that milling time and ball size affected the size of nano chitosan produced. The longer time milling, the smaller size of chitosan obtained. In addition, ball size also affected the nano chitosan produced. It is clearly known that small ball size ( 3 $\mathrm{mm}$ ) decreased the particle size bigger than the $6 \mathrm{~mm}$ ball. It was revealed that the use of 3 $\mathrm{mm}$ milling balls gives the maximum grinding effect because the chances of the ball pounding the powder are getting bigger.

Decreasing particle size is caused by mechanical deformation during the mechanical milling process. During this process collisions occurred between milling balls, chitosan powder and vial walls that occurred continuously so that the collision will produce collision energy that can reduce the size. When two colliding balls there are small amounts of powder trapped between the two balls and that happens repeatedly. During the milling process, powder particles will undergo a process of cold welding and repeated destruction. The surface of the newly formed mixed powder particles allows the cold back welding process to occur between fellow particles to form a new particle that is larger than the original size. Then the particles will again experience collisions and eventually return to destruction, and so on until they reach nano size [12].

The use of milling balls with different diameters $(3 \mathrm{~mm}$ and $6 \mathrm{~mm}$ ) turns out to give a more maximal grinding effect because the larger the possibility of contaminants will be bigger, even though the energy that will be produced is also large but the ball that will pound the powder will be smaller. Whereas if you use a small milling ball all then the energy produced is also small, but the process of milling can be more maximal [13].

\subsection{Zeta potential}

Zeta potential is a general measure of the amount of electrostatic charge of particles in dispersion, and is very suitable in the study of the stability of nanoparticle suspensions. The zeta potential is used to characterize the surface properties of nanoparticles and is also 
needed to determine the stability of nanoparticles. The potential zeta value of nano chitosan can be seen in Figure 4.

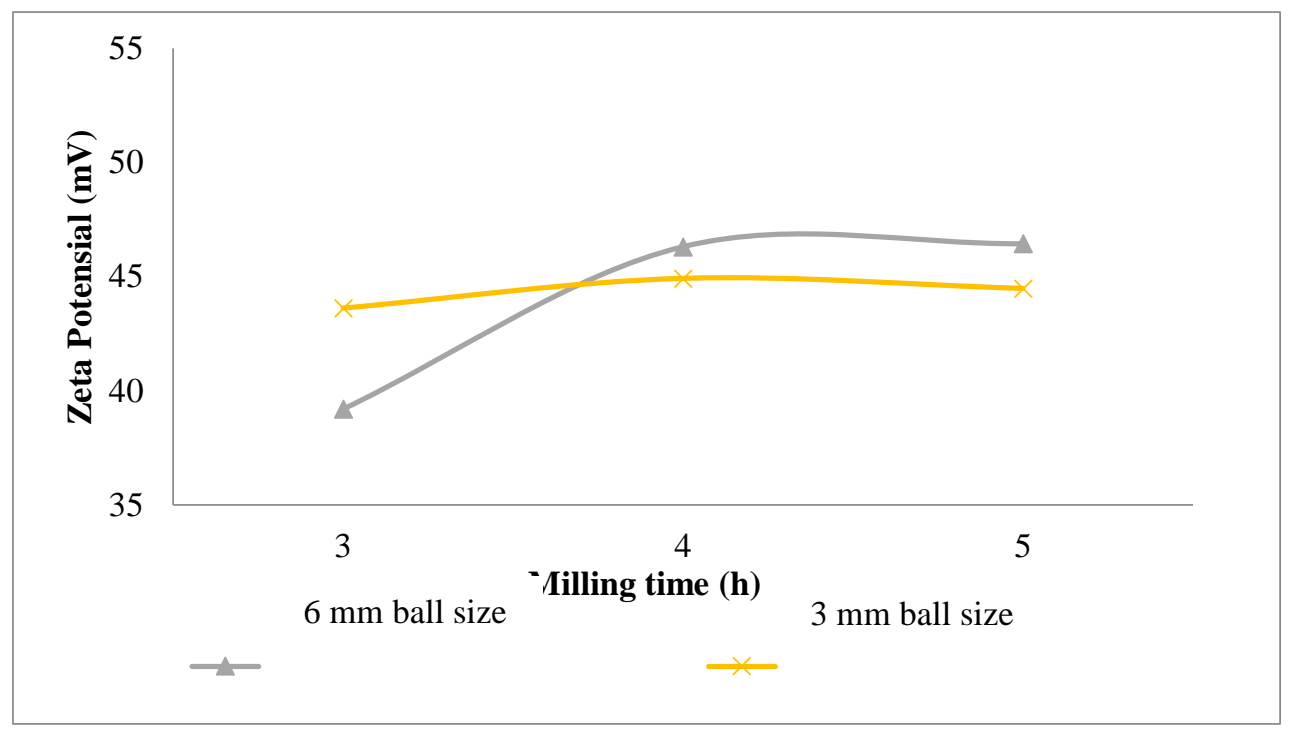

Fig. 4. Zeta Potential of nano chitosan at various treatments

The picture above can be seen that the zeta potential of nano chitosan ranges from $39.19 \mathrm{mV}-46.44 \mathrm{mV}$. The $3 \mathrm{~mm}$ and $6 \mathrm{~mm}$ milling balls factors relatively increase potential zeta values as the milling time increases. The colloidal system formed tends to be stable because it has a potential zeta value above (+) $30 \mathrm{mV}$. Nanoparticles that have a potential zeta above $\pm 40 \mathrm{mV}$ show a stable suspension, this is because surface loads will prevent aggregation between particles [14].

The results of the analysis showed that the milling time factor and the difference in the size of the milling balls did not have a significant effect on the zeta potential of nano chitosan produced. The relatively stable zeta value is thought to be caused by the use of tween 80 . The use of this surfactant is to reduce the tension between solid-liquid surfaces so that a repulsive force is generated between particles that blocks aggregation and also for maintaining stability [15].

\section{Conclusion}

The characteristics of the best chitosan nanoparticles with the top down method are 5 hours milling time with small milling balls $(3 \mathrm{~mm})$ with particle size values $248.28 \mathrm{~nm}$, potential zeta $44.92 \mathrm{mV}, 96 \%$ yield and viscosity $104.88 \mathrm{cP}$. It is known that small milling balls $(3 \mathrm{~mm})$ gave a smaller size of chitosan compared to the bigger milling ball $(6 \mathrm{~mm})$.

\section{Reference}

1. Mekawati F.E, Sumardjo D, Jurnal Sains And Matematika 1, 51-54 (2000)

2. Hargono, Abdullah, Sumantri I, Jurnal Reaktor 12, 53-57 (2008)

3. Tiyaboonchai W, Naresuan University Journal 11, 51-66 (2003) 
4. Safari J, Zarnegar Z, Jazini ZA, Sadeghi M, Catal. Commun. 77, 108 (2016)

5. Harahap Y, Universitas Indonesia, (2012)

6. Benjakula S, Sophanodora, Asean Food Jurnal 8, 145-148 (1993)

7. Yang MH, Yuan SS, Huang YF, Lin PC, Lu CY, Chung TW, Tyan YC. BioMed Research International 137, 1-9 (2014)

8. Sarimai S, Ratnawulan R, Ramli R. Fauz A. IOP Conf. Series: Materials Science and Engineering 335 (2018) 012004

9. Barbosa, Gustavo V, Enrique O.R, Pablo J, Hong Y, Kluwer Academic Plenum Publisher (2005)

10. Protan Laboratories, USA: Burgess (1989)

11. Mohanraj U.J, Chen Y, Tropical Journal of Pharmaceutical Research 5, 561-573 (2006)

12. Simanjuntak B.A, Purwaningsih H, Jurnal Teknik 1, 2301-9271 (2012)

13. Suryanarayana C, Colorado School of Mines Golden, (2003)

14. Avadi M.R, Assal M.M.S, Nasser M, Saideh A, Fatemeh A, Rassoul D, Mrteza R, Nanomedicine: Nanotechnology, Biology, and Medicine 6, 58-63 (2010)

15. Wahyuningsih I, Putranti W, Pharmacy 12,1-19 (2015) 